\title{
The History of Preconception Care: Evolving Guidelines and Standards
}

\author{
Margaret Comerford Freda • Merry-K. Moos • \\ Michele Curtis
}

Published online: 19 May 2006

(C) Springer Science+Business Media, Inc. 2006

\begin{abstract}
This article explores the history of the preconception movement in the United States and the current status of professional practice guidelines and standards. Professionals with varying backgrounds (nurses, nurse practitioners, family practice physicians, pediatricians, nurse midwives, obstetricians/gynecologists) are in a position to provide preconception health services; standards and guidelines for numerous professional organizations, therefore, are explored. The professional nursing organization with the most highly developed preconception health standards is the American Academy of Nurse Midwives (ACNM); for physicians, it is the American College of Obstetricians and Gynecologists (ACOG). These guidelines and standards are discussed in detail.
\end{abstract}

Keywords Preconception health · Women's health . Guidelines and standards

M. C. Freda $(\square)$

Department of Obstetrics \& Gynecology and Women's Health, Albert Einstein College of Medicine, Montefiore Medical Center, Bronx, NY

e-mail: margaretfreda@yahoo.com

M.-K. Moos

Department of Obstetrics and Gynecology, University of North Carolina,

Chapel Hill, NC

M. Curtis

University of Texas-Houston HSC, Attending physician,

LBJ Hospital

\section{Introduction}

From the earliest recorded time, women have been advised to increase their level of wellness and to avoid hazardous substances before becoming pregnant. Plutarch wrote that the ancient Spartans: “....ordered the maidens to exercise themselves with wrestling, running, throwing the quoit and casting the dart, to the end that the fruit they conceived might, in strong and healthy bodies, take firmer root and find better growth" [1]. In the Old Testament [2], the following passage appears: "And the angel of the Lord appeared unto the woman, and said unto her, 'Behold now thou art barren, and bearest not: but thou shalt conceive and bear a son. Now, therefore beware, I pray thee, and drink not wine and strong drink, and eat not any unclean thing." "

As the modern practice of obstetrics evolved, it became a specialty that separated women's prepregnancy wellness from prenatal care, but in the last quarter century this has begun to change. The dominant model of prenatal care as the main perinatal prevention strategy has been reexamined, and recognition of the importance of the prepregnancy period has emerged. Today the general public and health care professionals could easily be overwhelmed by the amount of information available which promotes prepregnancy or preconceptional health. A Google search (conducted April 25, 2005) of the term "preconception health" identified 630,000 hits. On the same day a search of the National Library of Medicine's PubMed found 338 entries for "preconception health" with the earliest published in 1978, and 103 articles for "preconceptional" with the earliest published in 1982; in addition, most of the articles identified through the two search terms were unique. The nation's approach to women's health care may well be at the tipping point of redefining the perinatal period to include women's wellness across the reproductive life span as an appropriate 
and favored approach to impacting reducing poor pregnancy outcomes.

Stimulated by research findings which underscored the limitations of traditional prenatal care on decreasing the incidence of congenital anomalies, leaders in the health community, professional organizations, advocacy groups and federal government began actively encouraging redefinition of the United States' perinatal prevention paradigm in the early 1980s. Because the usual pathways to impact on perinatal outcomes (prenatal and neonatal care) often start too late to achieve primary prevention, the need to reach women with prevention opportunities before prenatal care was recognized and the concept of preconceptional health promotion emerged. This article explores the history of the preconception movement in the United States and the current status of professional practice guidelines and standards.

\section{The decade of the eighties}

One of the first federal position papers to acknowledge the need to change the nation's approach to prevention appeared in 1979; while focused on the need to develop a continuum of child health care, it included the concept of prepregnancy care [3]. The programmatic elements in the proposed comprehensive package of integrated services were identified as:

- Interconceptional care

- Prenatal care

- Perinatal care

- Child Health care

- Services for handicapped children

- Adolescent services

Interest in moving away from categorical care to more integrated and comprehensive services has subsequently become a hallmark of current efforts to redefine the perinatal prevention paradigm. Why the list employed the word "interconceptional" rather than preconceptional is not clear. Since 1979, "interconceptional" care has come to represent efforts to address health status between pregnancies, birth spacing and intendedness of subsequent conceptions; "preconceptional" generally refers to the woman's health status and risks before the first pregnancy and her health status shortly before any conception. "Periconceptional" usually refers to the time immediately before conception through the period of organogenesis. It is important to note that this nomenclature is inconsistently applied and not universally accepted. In England, for instance, "preconceptional" is referred to as "preconceptual."

By 1985, the concept of preconceptional care and its potential advantages began to gain momentum. In that year, the Institute of Medicine published Preventing Low Birthweight [4] and noted that numerous opportunities exist before preg- nancy to reduce the incidence of low birth weight, but that they are too often overlooked in favor of interventions during pregnancy. The Committee emphasized the importance of prepregnancy risk identification, counseling and risk reduction; health education related to pregnancy outcome in general, and to low birthweight, in particular. The Committee supported restructuring the perinatal prevention paradigm by noting:

"Much of the literature about preventing low birthweight focuses on the period of pregnancy-how to improve the content of prenatal care, how to motivate women to reduce risky habits while pregnant, how to encourage women to seek out and remain in prenatal care. By contrast, little attention is given to opportunities for prevention before pregnancy. Only casual attention has been given to the proposition that one of the best protections available against low birthweight and other poor pregnancy outcomes is to have a woman actively plan for pregnancy, enter pregnancy in good health with as few risk factors as possibly, and be fully informed about her reproductive and general health." (p. 119)

The IOM Committee advocated that family planning services be positioned as an essential component of effective preconceptional initiatives, thereby supporting the integration of services rather than reinforcing firm boundaries of categorical programs. It also recommended that the content of reproductive health education, particularly in schools and family planning settings, be expanded to introduce concepts of prepregnancy wellness. The third recommendation of the Committee was to develop the notion of preconceptional consultation to identify and reduce risks associated with poor pregnancy outcomes, particularly for women who had already experienced a poor outcome.

In 1989 another federally appointed committee, The Expert Panel on the Content of Prenatal Care, gave a strong endorsement to preconceptional health when it recommended that the preconception visit may be the single most important health care visit when viewed in the context of its effect on pregnancy [5]. The Panel went on to suggest that the last family planning visit should be the preconception visit and that the concept of preconception care should become a routine part of prenatal care with accompanying reimbursement and coverage included in all health insurance plans. However, the Panel indicated that preconception care is likely to be most effective when services are provided as part of general preventive care or during primary care visits for medical conditions. This approach has come to be referred to as "opportunistic care" because it takes advantage of the opportunities afforded by visits for other reasons.

Specific components of preconception care were identified by the Panel as (1) risk assessment, (2) health promotion and (3) intervention and follow-up. The Panel advocated 
that the preconception assessment include a holistic range of investigations including: individual and social conditions, adverse health behaviors, medical, psychological and environmental conditions and barriers to family planning and early prenatal care enrollment.

In 1983, the American Academy of Pediatrics (AAP) and the American College of Obstetrics and Gynecologists (ACOG) in partnership with the March of Dimes Birth Defects Foundation published the first Guidelines for Perinatal Care [6]. Appendix C of those first guidelines notes that "Preparation for parenthood should begin prior to conception. At the time of conception the couple should be in optimal physical health and emotionally prepared for parenthood" (p. 257). The Guidelines indicated that when pregnancy is contemplated, the preconception visit should be part of a comprehensive gynecologic examination, and the history should include a detailed family history including ethnic background as well as investigation of lifestyle issues, religion, the home and work environments, hobbies, pets, immunizations, medications and dietary habits. The guidelines recommended use of an extensive checklist to systematically explore areas of potential significance. Based on the patient's history, the "counseling and instruction session" could be focused in a variety of directions including testing for genetically transmitted diseases and the parent's carrier status, as well as counseling regarding potential teratogenic exposures, exploration and interventions to address medical problems prior to pregnancy, interconceptional care to address the likelihood of recurrence of any complications experienced in previous pregnancies, including congenital anomalies, and the importance of recording menstrual dates to allow the earliest possible initiation of prenatal care.

In 1985, ACOG provided a small grant to Moos and Cefalo to develop and test a checklist which came to be known as the preconceptional health appraisal $[7,8]$. The appraisal was designed to aid providers in conducting comprehensive screening for preconceptional risks in a time-efficient manner and employed a self-assessment tool with built-in educational feedback. First introduced into local health department family planning clinics in North Carolina, it was adopted over the next several years by numerous state health departments across the country as well as private providers, large health maintenance organizations and several Canadian provinces. The commitment of public health organizations to introduce women to the concepts of preconceptional health during routine family planning visits placed the public sector in the position of being a leading innovator in the preconception movement. One state that demonstrated particular commitment to changing the perinatal prevention paradigm was Wisconsin. By the mid-1980's, the Wisconsin Association for Perinatal Care was calling for redefinition of peri- natal care to include the preconceptional period [9], and it has been unwavering in its commitment to improve pregnancy outcomes through preconceptional health promotion strategies ever since.

Concurrent with the above noted activities, additional credence to the benefits of preconceptional health promotion and primary prevention was provided when two nationally recognized obstetrician-gynecologists published books on the topic, one for the professional community and one for the consumer market $[7,10]$.

\section{The decade of the nineties}

Healthy People 2000, the national health promotion and disease prevention objectives for the United States, published in 1990, moved preconceptional care into a standard expectation within the health care system when it defined it as a one of its service and protection objectives. The specific objective reads:

- Increase to at least $60 \%$ the proportion of primary care providers who provide age-appropriate preconception care and counseling (p. 199)

Supporting rationale for the objective states that "the purpose of preconception care and counseling is to ensure that couples are healthy prior to pregnancy and prepared to assume the responsibilities of parenthood, thereby reducing the risk of poor pregnancy outcomes" (p. 199). The remainder of the rationale not only echoes the position of the earlier 1989 Expert Panel on the Content of Prenatal Care regarding the opportunities of preconception care to address many medical conditions, personal behaviors and environmental conditions [5] but also stresses preconceptional health promotion as an opportunity to educate young people about the risks of sexual activity, including unintended pregnancies, disease-related infertility and cancers [11]. In 1993, the March of Dimes Birth Defects Foundation published Toward Improving the Outcome of Pregnancy: The 90s and Beyond which introduced the concept of "reproductive awareness" as the basic health promotion strategy needed to improve pregnancy outcomes [12]. The document, often referred to as TIOP, reflects the position of the March of Dimes' Committee on Perinatal Health. Because awareness of reproductive risks, healthy behaviors and family planning options is essential to improving the outcome of pregnancy, the committee declared that "a society-wide change in reproductive awareness is needed in the United States" (p. 12) and called for "a new strategy to reach each woman of childbearing age with reproductive awareness messages at every health encounter"(p. 12). The committee called upon health care providers to move away from the expectation that the 
prevention of poor pregnancy outcomes is only the professional responsibility of obstetrical providers by noting:

"A woman's health status in relationship to pregnancy usually is not considered until the first prenatal visit, and prenatal care often is segregated from other health care by provider type and by payment mechanism. Yet women of childbearing age have many encounters with the health care system. These include visits to gynecologists, pediatricians, internists, family physicians, nurse midwives and nurse practitioners, in settings such as public health, school health, women's health, substance abuse treatment, family planning, and sexually transmitted disease clinics and private offices (p. 13).”

TIOP goes on to call for professional standards, structures and financing to be set in place to ensure an annual preconception or interconception risk reduction visit for every woman from menarche to menopause and that a prepregnancy planning visit become a standard component of maternity care, routinely available to all women and integrated into the perinatal care delivery system. TIOP also stressed that reproductive awareness is not sex or gender specific and that the need to reach out to males, especially during the preconception and interconception periods, requires thoughtful and innovative strategies.

ACOG published its first technical bulletin on preconception care in May, 1995 [13] The bulletin called for thorough and systematic identification of risks, the provision of education individualized to the patient's needs, and the initiation of any desired interventions. Risk identification was broadly defined to include the areas of medical, reproductive, and family histories, nutritional habits, drug and environmental exposures and social issues and these efforts were to be targeted at improving outcomes for both the mother and the fetus ACOG took the position that rather than ensuring healthy pregnancy outcomes as suggested by other position papers, preconception care allows the woman and her partner, if so desired, to make informed-decisions. It states:

"Once information is available, the patient can be informed about the certainty or limitations of available information, what the risks of pregnancy may be, and precautions that may be beneficial. After women have been informed of the increased risks pregnancy may pose to their health or the health of a fetus or both, they can accept the increased risks, choose to modify their risks or opt to avoid childbearing (p. 2)."

ACOG cautioned against over promising the benefits of preconceptional care to both providers and patients by specifically noting that preconceptional services do not guarantee good pregnancy outcomes. It noted that placing emphasis only on women who are planning their next conception or women who have been identified as high-risk will result in a significant number of missed opportunities for primary prevention. Women who experience an unintended pregnancy are at least as likely to have risk factors for poor pregnancy outcomes as women who consciously plan the timing of their pregnancy. Therefore, ACOG recommended that routine visits by women who may, at some time, become pregnant are important opportunities to emphasize the importance of preconceptional health and habits and the advantages of planned pregnancies. Finally, ACOG called for a coordinated multispecialty effort directed by the obstetrician-gynecologist as a means to provide a comprehensive framework for preconceptional health care for all women of childbearing potential.

\section{The current decade}

In 2002, the fifth edition of the AAP/ACOG Guidelines for Perinatal Care was published [14]. In the preceding editions, preconception care had moved from an appendix item to the main text in preceding editions. This latest edition, however, reflects a shift away from framing preconceptional care as appropriately targeted toward prospective parents who are contemplating pregnancy to an emphasis on integration of preconceptional health promotion into all health encounters during a woman's reproductive years.

While some efforts to operationalize a redefined perinatal prevention paradigm grew, others dropped back. For example, Healthy People 2010 eliminated preconceptional health as a specific objective [15]. Except for the objective, "Increase the proportion of pregnancies begun with an optimum folic acid level" (target: 80\%), the relevance of a woman's health status at the time of conception to pregnancy outcomes is not referenced in the document, and reliance on prenatal and neonatal care to impact on pregnancy outcomes is reinforced as the preferred strategy to meet the perinatal objectives.

\section{Evolving standards and guidelines from professional organizations}

As the concept of preconception care has evolved in the health care community, several professional organizations have addressed its importance for their members, and some have developed specific guidelines or standards for preconception care.

\section{Nursing organizations}

In nursing, there are several professional organizations whose members care for reproductive age women. The AANP (American Academy of Nurse Practitioners) is the 
largest national professional organization for nurse practitioners of all specialties, with 15,000 members. While many nurse practitioners might be delivering preconception health services, AANP has not developed specific educational standards specific to this topical area, nor do their generic practice standards include preconception health. According to AANP Executive Director Dr. Judith Dempster (personal communication, April 11, 2005), members with questions about preconception health standards would be referred to specialty organizations such as AWHONN.

AWHONN (Association of Women's Health, Obstetric and Neonatal Nurses) is the official professional organization for women's health and obstetric nurses, and is comprised of 22,000 nurses whose goals are to promote excellence in nursing practice. While AWHONN does not have specific standards of care for women during the preconception period, they do offer many products to their members which discuss preconception health, including a practice monograph which outlines the importance and components of preconception care [16]. They also publish a Clinical Position Statement which states that AWHONN supports legislation and policies that encourage women of childbearing age to consume 400 micrograms of synthetic folic acid every day [17]. In addition, AWHONN is also a member of the National Council on Folic Acid (NCFA), a partnership of over 80 national organizations and associations, state folic acid councils and government agencies. While not strictly a preconceptional project, AWHONN developed a specific Clinical Position Statement on Smoking and Childbearing, urging nurses to screen women for tobacco use and help women stop smoking [18]. Although nurses initiated this program during pregnancy, it had major interconceptional ramifications for the women involved, and has added to the literature on smoking cessation for women of reproductive age [19].

ACNM, the American College of Nurse Midwives is the professional organization for all nurse midwives in the United States, and represents over 7,000 nurse midwives who are providers of comprehensive women's health. In their Core Competencies for Basic Midwifery Practice (www.acnm.org/prof/display.cfm?id=137) they have specific educational and practice standards for preconception health for women. These standards are:

"V. Components of Midwifery Care: The Primary Health Care of Women

B. Applies knowledge of midwifery practice in the preconception period that includes, but is not limited to, the following:

1. Assessment of individual and family readiness for pregnancy, including emotional, psychosocial and sexual factors
2. Impact of health, family and genetic history on pregnancy outcomes

3. Influence of environmental and occupational factors, health habits, and behavior on pregnancy planning

4. Health and laboratory screening to evaluate the potential for a healthy pregnancy" (p. 3)

NAPNAP (the National Association of Pediatric Nurse Practitioners) does not publish any guidelines or standards for preconception care. Their members provide services for adolescents, but the organization has not yet addressed preconception care in a structured manner (personal communication Dr. Karen Kelly Thomas, Executive Director NAPNAP, April 13, 2005). They have, however, published at least one article in their official journal on the topic [20].

In terms of general nursing education, the AACN (The American Association of Colleges of Nursing) does not offer specific guidelines for what is to be taught to student nurses about preconception health, but rather sets standards for overall educational goals.

\section{Organizations for all professionals}

The March of Dimes, while not an organization which targets just one type of professional, has been producing numerous materials for health care professionals about preconception health for many years. Materials and products that the March of Dimes produces for providers of care include: their nursing module Preconception Health Promotion: A Focus for Women's Wellness [21], a Preconception Curriculum consisting of power point slides for Obstetricians, Family Medicine specialists, Pediatricians, and Internists which suggests that every visit to a health care provider should include elements of preconception care, a Preconception Screening and Counseling Tool, grants to the March of Dimes Chapters for preconception services, an educational program called Genetics and Your Practice (CD and online), and a Genetic screening pocket guide. Many of these can be found on their website www.marchofdimes.com, and their Spanish language website www.nacersano.org. They also produce an e-preconception newsletter (Spanish), an education program for Spanish women called Comenzando Bien, and pamphlets for patient education such as Are You Ready?, Think Ahead for a Healthy Baby, Folic Acid brochures, and Pre-Pregnancy Planning Fact Sheet.

The CDC, the March of Dimes, and the National Council on Folic Acid (NCFA) have organized a national folic acid promotion effort for the prevention of serious birth defects of the brain and spine (neural tube defects or NTDs). The goal of the effort is to teach all women about the importance of getting enough folic acid every day. The effort aims to reach every woman who could possibly 
become pregnant, as well as teach health care professionals and community advocacy groups about the importance of folic acid. The CDC, the March of Dimes, and NCFA have created messages, materials, websites, and other tools to reach providers and women with the folic acid message. In 2005, the CDC and March of Dimes collaborated to develop a national summit on preconception care in order to bring together all the stakeholders in the subject; this supplement issue of Maternal Child Health is one of the many consequences of these efforts by CDC/MOD.

\section{Physician organizations}

The American Academy of Pediatrics represents 60,000 pediatricians, many of whom provide adolescent services to young women of reproductive age. They publish, along with the American College of Obstetricians and Gynecologists, Guidelines for Perinatal Care, the document which is generally accepted to be the outline for acceptable care during the perinatal period for women and infants. In the current edition, 5 pages are devoted to preconception care, which is described as "the identification of those conditions that could affect a future pregnancy or fetus, and that may be amenable to intervention (p. 73)" [14]. The Guidelines note that all encounters with women of reproductive age should include counseling about health which could optimize pregnancy outcome. AAP is also a member of the National Organization on Fetal Alcohol Syndrome, which addresses medical school curriculum for fetal alcohol syndrome, and AAP supports numerous state-wide programs to enhance adolescent health before childbearing. In the American Board of Pediatrics study guide for the certifying examining for Adolescent Medicine certification, materials included are prevention of pregnancy, prevention of substance use, and general reproductive health care, all of which contribute to better preconception health (www.aap.org/sections/adol/adol.pdf).

The American Academy of Family Physicians (AAFP) represents 94,000 family physicians, residents and medical students in the United States, and sets the professional standards for care delivered by family physicians. While no specific guidelines or standards for preconception health services developed by AAFP could be located, AAFP addresses the issue of preconception care in many articles in their official journal, and in other practice venues such as suggestions to increase use of folic acid [22,23].

CREOG is the Council on Resident Education in Obstetrics and Gynecology, and is charged with setting the educational standards for obstetricians and gynecologists in training. CREOG's Educational Objectives are contained in its core curriculum [24]. Regarding preconception care, CREOG's core curriculum states: "In evaluating patients for preconceptional care, obstetrician gynecologists must assess those factors of the history, physical examination, and di- agnostic studies that pregnancy would alter; assess the patient's access to and compliance with a plan of prenatal care; and consult with or refer her to other experts on specific conditions that may arise during the pregnancy."(p. 39). Its standards are as follows:

\section{“ II. Antepartum Care-Preconceptional Care}

1. Perform a thorough history, assessing historical and ongoing risks that my affect future pregnancy

2. Counsel a patient regarding the impact of pregnancy on maternal medical conditions.

3. Counsel a patient regarding the impact of maternal medical conditions on pregnancy

4. Counsel a patient regarding appropriate lifestyle modification conducive to favorable pregnancy outcome.

5. Counsel a patient regarding appropriate preconception testing.

6. Counsel a patient regarding pregnancy associated risks and conditions, such as advanced age, hypertension, diabetes, genetic disorder, prior aneuploid or anomalous fetus/newborn" (p. 49)

ACOG, the American College of Obstetricians and Gynecologists represents 46,000 obstetricians and gynecologists in the United States, and in 2005 celebrated its 50th anniversary. ACOG has advocated for preconception care for several decades, and offers the most detailed guidelines for the provision of preconception services. ACOG's acknowledges that while ideal health prior to pregnancy does not guarantee a perfect or uncomplicated pregnancy, women who are considering pregnancy should undergo comprehensive preconception evaluation, examination, and counseling. However, preconception care is best when it is provided as part of primary health care, rather than as a discrete new category of health care, particularly since almost one-half of all pregnancies in the United States are unintended [25, 26].

\section{Components of preconception care-ACOG}

In examining multiple documents produced by ACOG in recent years, certain components of preconception care can be delineated.

\section{Periodic assessments}

Preconception counseling should occur at periodic assessments for all reproductively capable women. These assessments may be yearly or as appropriate [27]. Counseling should include discussions about the likelihood of pregnancy (planned or unplanned), counseling on appropriate medical care and behavior to optimize pregnancy outcomes, specific 
health risks the woman has that may predispose her to an adverse pregnancy outcome if not addressed, and how to either effectively prevent an unplanned pregnancy or to maximize her physical and emotional health prior to a planned or even unplanned event. Even clinicians that do not provide prenatal care should be able to provide preconception counseling and screening to their reproductively capable patients.

The patient's history should be reviewed and updated on an annual basis or more often if appropriate. The history should include a review of any medical conditions and medications the patient may be using. It is important to ask about prescription as well as over the counter drugs, herbs, and supplements. Inquiries about occupational and hobby exposures to chemicals, solvents, or heavy metals should be made. In this manner, potentially teratogenic agents may be identified.

The patient's reproductive history may provide important clues about future pregnancy risks. Recurrent miscarriages should raise suspicion of a possible genetic or chromosomal problem. The neonatal outcome of previous pregnancies should be noted but it is equally as important to ask women about the current health of any children they have as some congenital disorders do not become manifest until several months or even years after birth and may not be considered by the family to represent a congenital defect.

\section{Genetic disorders and genetic screening}

The woman's family history and ethnicity for genetic disorders and malformations should be obtained. Carrier screening for Tay-Sachs disease is recommended for individuals of Eastern European (Ashkenazi) Jewish, French Canadian and Cajun descent [28]. ACOG recommends offering cystic fibrosis screening to individuals with a family history, reproductive partners of persons with cystic fibrosis, and to couples in whom one or both partners are Caucasian and are planning pregnancy [29].

Carrier screening for Tay-Sachs, Canavan disease, cystic fibrosis, and familial dysautonomia should be offered to Ashkenazi Jewish individuals during preconception screening. These same individuals may be offered or may request screening for Gaucher disease, Niemann-Pick disease type A, Fanconi anemia group C, Bloom syndrome, or mucolipidosis IV as well. Carrier screening should be offered to any individual with a positive family history of one of these disorders; the screening should be for the specific disorder. When only one partner is of Ashkenazi Jewish descent, that individual should be screened first. Except for Tay-Sachs and cystic fibrosis, the carrier frequency and detection rate for the other disorders is unknown.

If an individual is found to be a carrier for Tay-Sachs or one of the other aforementioned diseases, their relatives are at risk for carrying the same mutation; carriers are encouraged to inform their relatives of the risk and availability of carrier screening [28].

Other screening tests exist for genetic disorders with an increased incidence in specific ethnic groups, e.g., alpha or beta thalassemia screening in Orientals, beta thalassemia screening for those of Mediterranean descent, and sickle cell disease screening for African-Americans.

Women who have had a previous pregnancy complicated by autosomal trisomy, a sex chromosome aneuploidy, or if either partner has a chromosome translocation or inversion or aneuploidy should be given education about their increased risk of recurrence in future pregnancies and a referral for pre-pregnancy genetic counseling should be offered [30].

\section{Immunizations and infectious diseases}

The woman's immunization status, history of childhood diseases, and risk for exposure to hepatitis, HIV, or other sexually transmitted infections needs to be updated at least annually. When practical, preconceptional immunization of women to prevent disease in the offspring is preferred to vaccination of pregnant women with certain vaccines [31].

Approximately $30 \%$ of US adults have serologic evidence of prior exposure to toxoplasma gondii, a known teratogen. Screening for Toxoplasma gondii is controversial because evidence that treatment prevents congenital disease is lacking. All reproductively capable women should be advised to avoid eating undercooked or raw meat, wear gloves when working with soil, and practice safe-handling techniques when changing cat litter [32].

Congenital cytomegalovirus (CMV) infection occurs in $1 \%$ of all live births in the United States and causes major neonatal illness in $5 \%$ to $10 \%$ of these cases. Women with young children or those who work with young children may be counseled about reducing the risk of CMV through universal precautions, e.g. the use of latex gloves and rigorous hand-washing after handling diapers or after exposure to respiratory secretions. There are currently no other specific recommendations as there is no evidence that screening and/or treatment programs prevent infection [32, 33].

All women should be kept current with age-appropriate vaccines. Reproductive aged women should be asked about previous infection with varicella and offered vaccination if they report no known history of chickenpox. Conception should be delayed until 1 month after the second injection is given. Women who expect to be at least 3 months pregnant during the influenza season (November to April) should be vaccinated.

\section{Behavioral/psychosocial factors}

A woman's lifestyle and social behaviors should be reviewed and updated frequently. The use of alcohol, smoking, 
or other substances may have significant effects on pregnancy. Smoking during pregnancy is associated with low birth weight, abruptio placenta, preeclampsia, and preterm labor [34]. A woman's personal situation has a significant effect on her health and often changes over time. Clinicians should ask all women about intimate partner violence and women should be aware that it may escalate during pregnancy. In addition, a woman's mental health history may provide clues about recurrence risks either during or after a pregnancy.

A woman's dietary habits should be reviewed. Daily folic acid intake of $0.4 \mathrm{mg}$ should begin at least 1 month before pregnancy and continue through the first trimester. For women who have had a child with a neural tube defect, a higher dose of folic acid $(4.0 \mathrm{mg} / \mathrm{d})$ is recommended and has been shown to decrease the recurrence rate of neural tube defects [35]. Women who may become pregnant—planned or unplanned-should be aware of recent FDA warnings to avoid specific types of fish as they may be high in methyl mercury - a known neurotoxin to a developing fetal nervous system. Women should also be questioned about the need for any dietary restrictions they may have required in childhood. Women with PKU in childhood may not currently be adhering to a phenylalanine free diet but hyperphenylananinemia is associated with neurological impairment in a developing fetus.

Maternal prepregnancy weight is associated with several adverse outcomes. Women weighing more than $>300 \mathrm{lbs}$ $(>136 \mathrm{~kg}$ ) prior to pregnancy have a markedly increased risk for developing gestational diabetes, preeclampsia, and requiring a cesarean delivery compared with women weighing 100-149 lbs (45-67 kg). In one study, even among a subsample of women who did not have any diabetic or hypertensive diseases, excess weight significantly increased the likelihood of macrosomia and NICU treatment [36]. The relationship between maternal obesity and perinatal outcomes is necessary when discussing routine care as well as specific preconception planning.

\section{Medical history and conditions}

While most reproductive aged women are healthy, there are specific medical conditions associated with adverse pregnancy outcomes if untreated or treated poorly. Some medical conditions are contraindications to pregnancy. Hypertension should be under control prior to initiation of a pregnancy and women using angiotensin-converting enzyme inhibitors and angiotensin II receptor antagonists who are considering pregnancy should be switched to other agents and adjusted on these medications prior to conception. Certain cardiac disorders, such as primary pulmonary hypertension, place women at a very high risk for morbidity or mortality during preg- nancy [37]. There is an increased risk (3-10\%, depending on the parent's condition) of congenital heart disease among children born to women who have a history of a congenital heard defect [38].

Women with a history of thromboembolism have an increased risk of recurrence during pregnancy. Women with a history of thrombosis should be offered testing for inherited or acquired thrombophilias, particularly if the results of the testing would affect the management of future pregnancies. Testing may be considered in women with a family history of thrombosis or a first degree relative with a specific mutation [39].

It is very important that women receive a thorough workup and evaluation prior to being diagnosed with a seizure disorder and started on medication. Women diagnosed with seizures of unknown etiology should be told that even if they are on no medications at all, they have a two-fold higher risk for congenital malformations in their offspring than individuals without seizures. Anticonvulsant therapy use during pregnancy, particularly hydantoin or valproic acid, is associated with an increased risk for specific congenital defects. It is important to determine if anticonvulsant medication can be safely discontinued or to initiate therapeutic changes before pregnancy occurs. Women should be told that enzyme-inducing antiepileptic drugs may lower the efficacy of combination oral contraceptives, so an oral contraceptive containing a higher estrogenic concentration may be necessary. For women taking antiepileptic drugs who are considering a pregnancy, folic acid supplementation $5 \mathrm{mg} /$ day is recommended for one month prior to conception and until the end of the first trimester [40].

Women with poorly controlled asthma before pregnancy tend to have increased difficulty with asthma during pregnancy [41]. Severe, poorly controlled asthma may have an adverse effect on fetal outcome as a result of chronic or intermittent maternal hypoxaemia [42].

Women with quiescent autoimmune disease or a distant history of disease should be carefully evaluated and counseled about maternal and fetal risks. Patients should be counseled that the best time to attempt conception is during periods of inactive disease.

Women should be made aware of the deleterious effects of hypothyroidism in pregnancy, including an increased incidence of abortion, obstetric complications and fetal abnormalities in untreated women [43, 44]. Women being treated for hypothyroidism will require increased doses of thyroxine early and throughout pregnancy in order to maintain adequate levels; this is especially important during the first trimester [45]. Low maternal thyroxine concentrations may lead to impaired neurodevelopmental outcome in the neonate and child. Routine assessment for the presence of subclinical hypothyroidism is not recommended. ACOG recommends 
testing of thyroid function only in women with a personal history of thyroid disease or symptoms of thyroid disease [46].

Diabetes mellitus represents a medical condition where preconception control has been proven to translate into improved maternal-fetal outcomes. Patients with poor glucose control during the first 8-10 weeks of pregnancy have a 2-3 fold higher risk of birth defects in their offspring than patients without diabetes. Good glycemic control before conception diminishes the risk of birth defects to a level comparable to that of the normal population [47].

Women should be asked about any ongoing dermatologic therapy she may be receiving. Isotretinoin (Accutane) is used for a variety of skin conditions and is a known teratogen. Some anti-psoriasis treatments (Acetretin $\mathrm{X}$ and methotrexate) are known teratogens, while some antiviral medications (podophyllum) are contraindicated in pregnancy.

Table 1 includes suggestions for the clinician during routine or periodic health assessments to optimize possible future pregnancy outcomes for all reproductively capable women.

Table 1 Suggestions for the clinician during routine or periodic health assessments to optimize possible future pregnancy outcomes for all reproductively capable women

- Determine if the woman suffers from any undiagnosed or uncontrolled medical problems. If she does, provide recommendations for treatment of these conditions and when it would be best to attempt pregnancy.

- Make sure the patient is aware of any associations between the medical condition(s) and medications(s) she is taking and their impact on pregnancy outcomes.

- Ask the woman about her reproductive intentions at every visit ascertain what her risk of an unplanned pregnancy may be; for women not actively seeking to become pregnant, discuss her current contraceptive method and any concerns or problems she may be having with it.

- Review the woman's family history-including new births among family members-annually as things change over time. Discuss any familial conditions that may herald an increased risk of adverse pregnancy outcome for the woman.

- Discuss the significance that nutrition can have on maternal fetal outcomes,e.g., the impact of $0.4 \mathrm{mg}$ folic acid per day on neural tube defects in women with no family or previous history of a neural tube defect), the need to avoid excessive vitamin usage, especially vitamins $\mathrm{A}$ and $\mathrm{D}$, and the additional measures women on restricted diets may need to take to optimize their health and the health of the developing fetus.

- Review the patient's social behaviors or lifestyle patterns-such as smoking, alcohol, or other substance use or abuse-that may affect pregnancy adversely and offer treatment options.

- Ascertain the immunity status of woman to rubella, hepatitis, and varicella. Ensure she is up to date on immunizations.

\section{Conclusion}

The concept of preconception care has gained momentum over the past two decades, and health care providers are increasingly being urged to provide such care by their professional organizations. It is hoped that, with its stated goal of improving birth outcomes, preconception care could help insure that the health status of all reproductively capable women would be optimized. In examining available standards and guidelines for preconception care, the professional nursing organization with the most highly developed preconception health standards is the American Academy of Nurse Midwives, and for physicians is the American College of Obstetricians and Gynecologists [48]. Other professional organizations examined did not have specific care standards for comprehensive preconception health, but have educated their members about one or more aspects of preconception health (smoking cessation, folic acid awareness).

\section{References}

1. Plutarch: Lycurgus 14 (trans. John Dryden). New York: Random House; 1932. p. 59-60.

2. Old Testament (Judges 13:3-4).

3. US Department of Health, Education and Welfare (PHS) HAS/BCHS. Primary care effectiveness-An approach to clinical quality assurance in BCHS programs and projects, Washington, DC: Author; January, 1979.

4. Institute of Medicine Committee to Study the Prevention of Low Birthweight. Preventing low birthweight. Washington, DC: National Academy Press; 1985.

5. U.S. Public Health Service Expert Panel on Prenatal Care. Caring for our future: the content of prenatal care. Washington, DC: Public Health Service. Department of Health and Human Services; 1989.

6. American Academy of Pediatrics/American College of Obstetricians and Gynecologists. Guidelines for perinatal care. Evanston, Illinois: American Academy of Pediatricians; 1983.

7. Cefalo RC, Moos MK. Preconceptional health promotion: a practical guide. Rockville, MD: Aspen; 1988.

8. Moos MK. Preconceptional health promotion: a health education opportunity for all women. Women Health 1989;5(3):55-67.

9. Leonard TA. Preconception: a much neglected aspect of prenatal care. Wis Med J 1987;86(1):213-17.

10. Queenan JT, Leslie KK (eds). Preconceptions: preparation for pregnancy. Boston, MA: Little, Brown and Company; 1989.

11. U.S. Department of Health and Human Services, Public Health Service. Healthy People 2000. DHHS Publication No (PHS) 9150212; 1990.

12. March of Dimes Birth Defects Foundation. Toward improving the outcome of pregnancy. White Plains, NY: March of Dimes Birth Defects Foundation; 1993.

13. American College of Obstetricians and Gynecologists. Preconception Care. ACOG Technical Bulletin number 205; May 1995.

14. American Academy of Pediatrics/American College of Obstetricians and Gynecologists. Guidelines for perinatal care. 5th ed. Elk Grove Village, IL: American Academy of Pediatricians; 2002.

15. U.S. Department of Health and Human Services. Healthy people 2010. McLean Va: International Medical Publishing, Inc; 2000. 
16. Hobbins D. Preconception care: maximizing the health of women and their newborns. Association of Women's Health, Obstetric and Neonatal Nurses. Washington, DC; 2001.

17. AWHONN. Preconceptional use of folic acid. Association of Women's Health, Obstetric and Neonatal Nurses. Washington DC: Author; 1999.

18. Albrecht SA, Maloni JA, Thomas KK, Jones R, Halleran J, Osborne J. Smoking cessation counseling for pregnant women who smoke: scientific basis for practice for AWHONN's SUCCESS project. J Obstet Gynecol Neonatal Nurs 2004 May-Jun;33(3):298305.

19. Albrecht SA, Maloni JA. SUCCESS Program. Presentation at annual convention Association of Women's Health, Obstetric and Neonatal Nurses. Tampa, Florida; June 2004.

20. Gottesman MM. Preconception education: caring for the future. $J$ Pediatr Health Care 2004;18(1):40-4

21. Moos MK. Preconceptional health promotion. Nursing module. New York: March of Dimes Birth Defects Foundation; 2003.

22. Brundage SC. Preconception health care. Am Fam Physician 2002;65(12). Available at ::http://www.aafp.org/afp/20020615/ 2507.html. Accessed May 9, 2005.

23. Kirkham C, Harris S, Grzybowski S. Evidence-based prenatal care: Part I. General prenatal care and counseling issues. Am Fam Physician 2005;71(7). Available at: http://www.aafp.org/afp/ 20050401/1307.html. Accessed May 9, 2005.

24. Council on Resident Education in Obstetrics and Gynecology. Educational objectives: core curriculum in obstetrics and gynecology. 8th ed. Washington, DC: Author; 2005.

25. Forrest JD. Epidemiology of unintended pregnancy and contraceptive use. Am J Obstet Gynecol 1994;170:1485-9.

26. Moos MK. Preconceptional wellness as a routine objective for women's health care: an integrative strategy. J Obstet Gynecol Neonatal Nurs 2003;32:550-6.

27. American College of Obstetricians and Gynecologists. Primary and preventive care: periodic assessments. Washington, DC: American College of Obstetricians and Gynecologists; 2003.

28. American College of Obstetricians and Gynecologists. Prenatal and preconceptional carrier screening for genetic diseases in individuals of eastern european jewish descent. Washington, DC: American College of Obstetricians and Gynecologists; 2004.

29. America College of Obstetricians and Gynecologists, American College of Medical Genetics. Preconception and prenatal carrier screening for cystic fibrosis: clinical and laboratory guidelines. Washington, DC: American College of Obstetricians and Gynecologists; 2001.

30. American College of Obstetricians and Gynecologists. Prenatal diagnosis of fetal chromosomal abnormalities. Washington, DC: American College of Obstetricians and Gynecologists; 2001.
31. American College of Obstetricians and Gynecologists. Immunization during pregnancy. Washington, DC: America College of Obstetricians and Gynecologists; 2003.

32. American College of Obstetricians and Gynecologists. Perinatal viral and parasitic infections. Washington, DC: American College of Obstetricians and Gynecologists; 2000.

33. Allaire AD, Cefalo RC. Preconceptional health care model. Eur J Obstet Gynecol Reprod Biol. 1998;78:163-8.

34. Morrison EH. Periconception care. Prim Care 2000;27:1-12.

35. Honein MA, Paulozzi LJ, Mathews TJ, Erickson JD, Wong LY. Impact of folic acid fortification of the US food supply on the occurrence of neural tube defects. JAMA 2001;285(23):2981-6.

36. Rosenberg TJ, Garbers S, Chavkin W, Chiasson M. Prepregnancy weight and adverse perinatal outcomes in an ethnically diverse population. Obstet Gynecol 2003;102(5, Part 1):1022-7.

37. Whittemore R, Hobbins J, Engle M. Pregnancy and its outcome in women with and without surgical treatment of congenital heart disease. Am J Cardiol 1982;50:641-51.

38. Romano-Zelekha O, Hirsh R, Blieden L, et al. The risk for congenital heart defects in offspring of individuals with congenital heart defects. Clin Genet 2001;59:325-9.

39. American College of Obstetricians and Gynecologists. Thromboembolism in pregnancy. Washington, DC: American College of Obstetricians and Gynecologists; 2000.

40. Morrell MJ. Guidelines for the care of women with epilepsy. Neurology 1998;51(suppl 4):S21-7.

41. Abbott J. Medical illness during pregnancy. Emerg Med Clin North Am 1994;12:115-28.

42. Demissie K, Breckenridge MB, Rhoads GG. Infant and maternal outcomes in the pregnancies of asthmatic women. Am J Respir Crit Care Med 1998;158:1091-5.

43. Lazarus JH. Treatment of hyper- and hypothyroidism in pregnancy. J Endocrinol Invest 1993;16:391-6.

44. Casey B, Dashe J, Wells E, McIntire D, Byrd W, Leveno K, Cunningham G. Subclinical hypothyroidism and pregnancy outcomes. Obstet Gynecol 2005;105(2):239-45.

45. Mandel SJ, Reed Larsen P, Seeley EW, Brent, GA. Increased need for thyroxine during pregnancy in women with primary hypothyroidism. NEJM 1990;323:91-6.

46. American College of Obstetricians and Gynecologists. Thyroid disease in pregnancy. ACOG Practice Bulletin No. 37. Obstet Gynecol 2002;100:387-96.

47. Kitzmiller JL, Gavin LA, Gin GD, et al. Preconception care of diabetes: glycemic control prevents congenital anomalies. JAMA 1991;265:731-6.

48. American College of Obstetricians and Gynecologists. The importance of preconception care in the continuum of women's health care. Committee Opinion No. 313. Obstet Gynecol 2005;106(3):665-6. 